\title{
A Conservation Difference Scheme of Generalized Boussinesq Equation
}

\author{
Xiaoli Jiang and Xiaofeng Wang \\ School of Mathematics and Physics, Bohai University, Jinzhou, Liaoning Province 121013, China \\ Correspondence should be addressed to Xiaoli Jiang; jxls309@163.com
}

Received 26 February 2017; Revised 13 June 2017; Accepted 18 June 2017; Published 6 September 2017

Academic Editor: Pilar R. Gordoa

Copyright (c) 2017 Xiaoli Jiang and Xiaofeng Wang. This is an open access article distributed under the Creative Commons Attribution License, which permits unrestricted use, distribution, and reproduction in any medium, provided the original work is properly cited.

\begin{abstract}
We focus on the algorithm research of a class of six-order generalized Boussinesq equation. We use the finite difference method to discrete the Boussinesq equation. The discrete format with the law of energy conservation is deduced; stability and existence and good order of convergence properties are also derived. The efficiency of the proposed method is tested to numerical results that the convergence of space is of second-order and the conservation law of energy is verified very well for the energy difference.
\end{abstract}

\section{Introduction}

In this paper, we consider the following generalized Boussinesq equation:

$$
\begin{aligned}
u_{t t}-u_{x x}-u_{x x t}+u_{x x x x}+u_{x x x x t t} & =\left(u^{2}\right)_{x x}, \\
x & \times t \in(a, b) \times[0, T), \\
u(a, t) & =u(b, t)=0, \\
u_{x x}(a, t) & =u_{x x}(b, t)=0, \\
u(x, 0) & =u_{0}(x), \\
& u_{t}(x, 0)=u_{1}(x),
\end{aligned}
$$

where $u_{0}(x)$ and $u_{1}(x)$ are smooth functions. And problem (1)-(3) satisfies the law of energy conservation

$$
\begin{aligned}
E(t)= & \|u\|_{L_{2}}^{2}+\left\|u_{x}\right\|_{L_{2}}^{2}+\left\|\varphi_{x}\right\|_{L_{2}}^{2}+\left\|\varphi_{x x}\right\|_{L_{2}}^{2}+\left\|\varphi_{x x x}\right\|_{L_{2}}^{2} \\
& +\frac{2}{3} \int_{0}^{L} u^{3} d x=E(0),
\end{aligned}
$$

where $(\varphi)_{x x}=(u)_{t}$.

The motion of the small amplitude long wave can be affected by many factors such as terrain, water flow, and ground friction, which can lead to some nonlinear effects. The communication process of waves or swells off the coast from the deep sea to the shore of the ocean is the physical phenomena we often faced in the study of ocean problems; because of being affected by many factors, including the impact of changes in terrain, water, and ground friction, this process will issue nonlinear effects, such as shallow deformation, climbing waves, refraction, and diffraction, and have a great impact on the production and life of the human near the ocean. So it is very important to study these phenomena. The most widely used model which could describe the changing of offshore wave propagation in math is the system model $\mathrm{Bq}$; however, due to the fact that the affection of the nonlinear waves in shallow water is powerful, the form of the model is more complex, such as containing a number of higher-order dispersion. This increases the difficulty to the theoretical analysis and algorithm research of this model and it is a great challenge for solving the real-time wave problem.

Abbott et al. [1] studied the movement pattern of irregular waves in port by approximately calculating the system $\mathrm{Bq}$ in a larger area. By using DHI model based on a combination of waves $\mathrm{Bq}$ system, Tao [2] provided the slot calculation method of dynamic boundary to simulate waves perfectly. Madsen et al. [3] considered the phenomenon 
of fragmentation and circulation for regular and irregular waves. The widely used numerical methods in solving $\mathrm{Bq}$ systems are finite difference method, finite element method, and finite volume method. Finite difference method is to use a continuous solution of regional grid consisting of a finite number of discrete points instead of the continuous function and continuous solution of the region on the discrete variables used in the definition of the function on the grid to approximate. Use difference quotient to approximate derivative in original equations and boundary conditions, so we can use the algebra system to approximate instead original equations and boundary conditions; using a variety of iterative methods to solve this system can obtain the numerical solution of the discrete points for the original problem. Based on the finite difference, Peregrine [4] gives a one-dimensional approximate solution of the Bq system. For Bq system, Zhang and Tao [5] established a division and no division format and analyzed the order of convergence for the format and stability. Using predictor-corrector format, Hong and Zhang [6] search the numerical solution for any depth nonlinear Bq model, a discrete second-order accuracy form, and modified third-order accuracy of the format with Taylor expansion. Based on the finite difference, Abbott et al. [1] give the estimated correction format for the numerical solution of two-dimensional Bq system. Based on variable differential formats on different layers of time, Zhu and Hong [7] realized the numerical solution of $\mathrm{Bq}$ system for arbitrary depth. For improved IMBq system, we have not found any instability yet. To study the nature of the solution, mathematicians have proposed effective numerical format. On the basis of the implicit tight method, El-Zoheiry [8] proposed a threeiterative method for solving numerical format. Bratsos [9] designed an implicit finite difference scheme, which can achieve second-order accuracy. At the same time, to deal with the nonlinear interaction of IBq system, Bratsos [10] constructed a prediction correction format. The application of finite element method and finite volume method in studying $\mathrm{Bq}$ system numerical is many, the application of mixed finite volume method is very active, and we are not going to repeat here.

Many efficient numerical algorithms have been proposed for various Bq systems; see [1-10] and the references therein. But some of them ignore the nonlinear effects, lack the necessary theoretical proof, or make some errors between the real problem and the system model. Furthermore, the numerical study of conservation difference algorithm for nonlinear high-order dispersion Bq system is rare; the main reason is that there are no fixed maturity and methods to deal with higher derivatives and transform the nonlinear effects. Thus, it is very meaningful to research a numerical algorithm which can ensure energy conservation for $\mathrm{Bq}$ system. The contributions of this work are twofold: (1) constructing a conservative scheme based on undetermined coefficients using the finite difference method; it is proved theoretically that this scheme has good performance; for the discrete format in the existing Bq wave system with highorder dispersion and nonlinear source terms, the discrete method is to take difference transformation for each term in the system, while the theoretical properties of the numerical solution are not taken into consideration; however, for the $\mathrm{Bq}$ system in this paper, the conservation scheme has more strict requirement on each term; (2) presenting the finite difference method with undetermined coefficients for energy conservation format, taking proper treatment for high-order dispersion and nonlinear source differential forms, and finally analyzing the above method theoretically. The results showed that with this method the differential form with complex structure can be found effectively, and it has good convergent order, which contributes to the establishment of the stability properties of the solutions.

\section{Commonly Used Symbols and Lemmas}

In this paper, we consider a new conservation difference scheme for problem (1)-(3). For convenience, we illustrate the following symbols at first:

$$
\begin{aligned}
& x_{j}=j h+a, \\
& t_{n}=n \tau,
\end{aligned}
$$

$$
0 \leq j \leq J=\frac{(b-1)}{h}, 0 \leq n \leq N=\frac{T}{\tau},
$$

where $u_{j}^{n}$ stands for the approximate value at the point of $\left(x_{j}, t_{n}\right), Z_{h}^{0}=\left\{u=u_{j} \mid u_{0}=u_{J}=0, j=0,1,2, \ldots, J\right\}$, and $C$ is the general constant (it has different values in different places).

We introduce the following difference symbols:

$$
\begin{aligned}
\left(u_{j}^{n}\right)_{x} & =\frac{u_{j+1}^{n}-u_{j}^{n}}{h}, \\
\left(u_{j}^{n}\right)_{\bar{x}} & =\frac{u_{j}^{n}-u_{j-1}^{n}}{h}, \\
\left(u_{j}^{n}\right)_{t} & =\frac{u_{j}^{n+1}-u_{j}^{n}}{\tau}, \\
\left(u_{j}^{n}\right)_{\bar{t}} & =\frac{u_{j}^{n}-u_{j}^{n-1}}{\tau}, \\
\bar{u}_{j}^{n} & =\frac{u_{j}^{n+1}+u_{j}^{n-1}}{2}, \\
\left\langle u^{n}, v^{n}\right\rangle & =h \sum_{j=0}^{J} u_{j}^{n} v_{j}^{n}, \\
\left\|u^{n}\right\|^{2} & =\left(u^{n}, u^{n}\right), \\
\left\|u^{p}\right\|_{p}^{p} & =h \sum_{j}^{J}\left|u_{j}^{n}\right|^{p}, \\
\left\|u^{n}\right\|_{\infty} & =\sup _{1 \leq j \leq J}\left|u_{j}^{n}\right| .
\end{aligned}
$$

Lemma 1 (see [11]). For any two mesh functions $u, v \in Z_{h}^{0}$, one has 
(1) $\left(u_{x}^{n}, v^{n}\right)=-\left(u^{n}, v_{\bar{x}}^{n}\right)$;

(2) $\left(u_{x \bar{x}}^{n}, v^{n}\right)=-\left(u_{x}^{n}, v_{x}^{n}\right)=\left(u^{n}, v_{x \bar{x}}^{n}\right)$;

(3) $\left(u_{x \bar{x}}^{n}, u^{n}\right)=-\left\|u_{x}^{n}\right\|^{2}$;

(4) furthermore, if $\left(u_{0}^{n}\right)_{x \bar{x}}=\left(u_{J}^{n}\right)_{x \bar{x}}=0$, then $\left(u_{x x \bar{x} \bar{x}}^{n}, u^{n}\right)=$ $\left\|u_{x x}^{n}\right\|^{2}$.

Lemma 2 (see [11] (discrete Sobolev's inequality)). For any discrete function $\left[X_{L}, X_{R}\right]$ on the finite interval $\left\{u_{j}^{n} \mid j=0,1\right.$, $2, \ldots, J\}$, there is the inequality

$$
\left\|u^{n}\right\|_{\infty} \leq C_{0} \sqrt{\left\|u^{n}\right\|} \sqrt{\left\|u_{x}^{n}\right\|+\left\|u^{n}\right\|}
$$

or

$$
\left\|u^{n}\right\|_{\infty} \leq \varepsilon\left\|u_{x}^{n}\right\|+C(\varepsilon)\left\|u^{n}\right\|
$$

where $C_{0}, \varepsilon$, and $C(\varepsilon)$ are three constants independent of $u_{j}^{n} \mid$ $j=0,1, \ldots, J$ and step length $h, \varepsilon$ can be any small, and $C(\varepsilon)$ is a constant dependent on $\varepsilon$.

Lemma 3 (see [12]). Suppose that $g(x) \in C^{2}\left[d_{1}, d_{2}\right]$ and $a_{1}, a_{2}, b_{1}, b_{2} \in\left[d_{1}, d_{2}\right]$. Then there are $\theta \in(-1,1)$ and $\eta \in$ $\left[d_{1}, d_{2}\right]$ such that

$$
\begin{aligned}
\frac{g\left(a_{2}\right)}{a_{2}-a_{1}}-\frac{g\left(a_{1}\right)}{b_{2}-b_{1}} \\
=g^{\prime}\left(\frac{1-\theta}{2} a_{1}+\frac{1+\theta}{2} a_{2}\right) \\
\quad-g^{\prime}\left(\frac{1-\theta}{2} b_{1}+\frac{1+\theta}{2} b_{2}\right) \\
=g^{\prime \prime}(\eta)\left(\frac{1-\theta}{2}\left(a_{1}-b_{1}\right)+\frac{1+\theta}{2}\left(a_{2}-b_{2}\right)\right) .
\end{aligned}
$$

Lemma 4 (see [11] (discrete Gronwall's inequality)). Suppose that the discrete functions $w(k), \rho(k)$ are nonnegative; $\rho(k)$ is nondecreasing, so that if nonnegative constants $c>0$ and $\forall k$, one can get

$$
w(k) \leq \rho(k)+c \tau \sum_{l=0}^{k-1} w(l),
$$

then $w(k) \leq \rho(k) e^{c \tau k}$.

Lemma 5 (see [13] (Brouwer fixed point theorem)). Let $H$ be a finite dimensional inner product space; suppose that $w: H \rightarrow$ $H$ is continuous and there exists $\alpha>0$ such that $(w(x), x)>$ $0 \forall x \in H$ with $\|x\|=\alpha$. Then there exists $x^{*} \in H$, such that $w\left(x^{*}\right)=0$ and $\left\|x^{*}\right\| \leq \alpha$.

\section{Difference Scheme and Its Discrete Conservative Laws}

We establish the three layers finite difference schemes based on the problem of (1)-(3), as follows:

$$
\begin{aligned}
& \left(u_{j}^{n}\right)_{t \bar{t}}-\left(\bar{u}_{j}^{n}\right)_{x \bar{x}}-\left(u_{j}^{n}\right)_{x \bar{x} t \bar{t}}+\left(\bar{u}_{j}^{n}\right)_{x x \bar{x} \bar{x}}+\left(u_{j}^{n}\right)_{x x \bar{x} \bar{x} t \bar{t}} \\
& =\frac{1}{3}\left(\left(u_{j}^{n+1}\right)^{2}+\left(u_{j}^{n+1}\right)\left(u_{j}^{n-1}\right)+\left(u_{j}^{n-1}\right)^{2}\right)_{x \bar{x}} \\
& u_{0}^{n}=u_{J}^{n}=0 \\
& \left(u_{0}^{n}\right)_{x \bar{x}}=\left(u_{J}^{n}\right)_{x \bar{x}}=0 \\
& u_{j}^{0}=u_{0}\left(x_{j}\right) \\
& \left(u_{j}^{0}\right)_{\bar{t}}=u_{1}\left(x_{j}\right) .
\end{aligned}
$$

Obviously, (11) requires two initial values to stat the iteration. Thirst value is obtained from the initial condition in (3). The second value can be approximated by Taylor's expansion about $t_{0}$

$$
u(x, \tau)=u(x, 0)+\tau u_{t}(x, 0)+O\left(\tau^{2}\right),
$$

where

$$
\begin{gathered}
u(x, 0)=u_{0}(x), \\
u_{t}(x, 0)=u_{1}(x),
\end{gathered}
$$

so that we can get $u_{j}^{1}=u\left(x_{j}, \tau\right)$. At that time, when $j=0$, it follows from (13) that

$$
\frac{1}{h^{2}}\left(u_{-1}^{n}-2 u_{0}^{n}+u_{1}^{n}\right)=\frac{1}{h^{2}}\left(u_{J-1}^{n}-2 u_{J}^{n}+u_{J+1}^{n}\right)=0
$$

then we define $u_{-2}^{n}=u_{-1}^{n}=u_{J+1}^{n}=u_{J+2}^{n}=0$. For convenience, the last term of (11) is defined by

$$
\begin{aligned}
& P\left(u^{n+1}, u^{n-1}\right) \\
& \quad=\frac{1}{3}\left(\left(u_{j}^{n+1}\right)^{2}+\left(u_{j}^{n+1}\right)\left(u_{j}^{n-1}\right)+\left(u_{j}^{n-1}\right)^{2}\right)_{x \bar{x}} .
\end{aligned}
$$

Theorem 6. The finite difference scheme (11)-(15) admits the following invariant:

$$
\begin{aligned}
E^{n}= & \frac{1}{2}\left(\left\|u^{n+1}\right\|^{2}+\left\|u^{n}\right\|^{2}+\left\|u_{x}^{n+1}\right\|^{2}+\left\|u_{x}^{n}\right\|^{2}\right) \\
& +\left\|\varphi_{x}^{n+1 / 2}\right\|^{2}+\left\|\varphi_{x x}^{n+1 / 2}\right\|^{2}+\left\|\varphi_{x x x}^{n+1 / 2}\right\|^{2} \\
& +\frac{1}{3} \sum_{j=1}^{J}\left(\left(u_{j}^{n+1}\right)^{3}+\left(u_{j}^{n}\right)^{3}\right)=E^{n-1}=\cdots=E^{0},
\end{aligned}
$$

where $\varphi_{j}^{n}$ satisfies $\left(\varphi_{j}^{n+1 / 2}\right)_{x \bar{x}}=\left(u_{j}^{n}\right)_{t}$.

Proof. Multiplying (11) by $\left(\varphi^{n+1 / 2}+\varphi^{n-1 / 2}\right)$, summing over $j$, we obtain

$$
\mathrm{I}-\mathrm{II}-\mathrm{III}+\mathrm{IV}+\mathrm{V}=\mathrm{VI},
$$


where

$$
\begin{aligned}
& \begin{aligned}
\mathrm{I} & =\left\langle\left(u^{n}\right)_{t \bar{t}},\left(\varphi^{n+1 / 2}+\varphi^{n-1 / 2}\right)\right\rangle=-\frac{1}{\tau}\left(\left\|\varphi_{x}^{n+1 / 2}\right\|^{2}\right. \\
& \left.-\left\|\varphi_{x}^{n-1 / 2}\right\|^{2}\right)
\end{aligned} \\
& \mathrm{II}=\left\langle\left(\bar{u}^{n}\right)_{x \bar{x}},\left(\varphi^{n+1 / 2}+\varphi^{n-1 / 2}\right)\right\rangle=\frac{1}{2 \tau}\left(\left\|u^{n+1}\right\|^{2}\right. \\
& \left.-\left\|u^{n-1}\right\|^{2}\right) \\
& \mathrm{III}=\left\langle\left(\bar{u}^{n}\right)_{x \bar{x} \bar{t} t},\left(\varphi^{n+1 / 2}+\varphi^{n-1 / 2}\right)\right\rangle=\frac{1}{\tau}\left(\left\|\varphi_{x x}^{n+1 / 2}\right\|^{2}\right. \\
& \left.-\left\|\varphi_{x x}^{n-1 / 2}\right\|^{2}\right) \\
& \mathrm{IV}=\left\langle\left(\bar{u}^{n}\right)_{\mathrm{x} x \bar{x} \bar{x}},\left(\varphi^{n+1 / 2}+\varphi^{n-1 / 2}\right)\right\rangle=-\frac{1}{2 \tau}\left(\left\|u_{x}^{n+1}\right\|^{2}\right. \\
& \left.-\left\|u_{x}^{n-1}\right\|^{2}\right) \\
& \mathrm{V}=\left\langle\left(u^{n}\right)_{x x \bar{x} \bar{x} \bar{t}},\left(\varphi^{n+1 / 2}+\varphi^{n-1 / 2}\right)\right\rangle=-\frac{1}{\tau}\left(\left\|\varphi_{x x x}^{n+1 / 2}\right\|^{2}\right. \\
& \left.-\left\|\varphi_{x x x}^{n-1 / 2}\right\|^{2}\right) \text {, } \\
& \mathrm{VI}=\left\langle P\left(u^{n+1}, u^{n-1}\right),\left(\varphi^{n+1 / 2}+\varphi^{n-1 / 2}\right)\right\rangle \\
& =\frac{1}{3}\left\langle\left(\left(u^{n+1}\right)^{2}+\left(u^{n+1}\right)\left(u^{n-1}\right)+\left(u^{n-1}\right)^{2}\right)_{x \bar{x}},\right. \\
& \left.\left(\varphi^{n+1 / 2}+\varphi^{n-1 / 2}\right)\right\rangle=\frac{1}{3}\left\langle\left(\frac{\left(u^{n+1}\right)^{3}-\left(u^{n-1}\right)^{3}}{u^{n+1}-u^{n-1}}\right)_{x \bar{x}},\right. \\
& \left.\left(\varphi^{n+1 / 2}+\varphi^{n-1 / 2}\right)\right\rangle=\frac{1}{3}\left\langle\left(\frac{\left(u^{n+1}\right)^{3}-\left(u^{n-1}\right)^{3}}{u^{n+1}-u^{n-1}}\right),\right. \\
& \left.\left(\varphi^{n+\frac{1}{2}}+\varphi^{n-\frac{1}{2}}\right)_{x \bar{x}}\right\rangle \\
& =\frac{1}{3}\left\langle\left(\frac{\left(u^{n+1}\right)^{3}-\left(u^{n-1}\right)^{3}}{u^{n+1}-u^{n-1}}\right),\left(u^{n}-u^{n-1}\right)_{t}\right\rangle \\
& =\frac{1}{3 \tau}\left\langle\left(\frac{\left(u^{n+1}\right)^{3}-\left(u^{n-1}\right)^{3}}{u^{n+1}-u^{n-1}}\right),\left(u^{n+1}-u^{n}\right)\right. \\
& \left.+\left(u^{n}-u^{n-1}\right)\right\rangle=\frac{1}{3}\left\langle\left(\frac{\left(u^{n+1}\right)^{3}-\left(u^{n-1}\right)^{3}}{u^{n+1}-u^{n-1}}\right),\right. \\
& \left.\left(u^{n+1}-u^{n-1}\right)\right\rangle=\frac{h}{3 \tau} \\
& \cdot \sum_{j=1}^{J}\left(\frac{\left(u_{j}^{n+1}\right)^{3}-\left(u^{n-1}\right)^{3}}{u_{j}^{n+1}-u_{j}^{n-1}}\left(u_{j}^{n+1}-u_{j}^{n-1}\right)\right)=\frac{h}{3 \tau} \\
& \cdot \sum_{j=1}^{J}\left(\left(u_{j}^{n+1}\right)^{3}-\left(u_{j}^{n-1}\right)^{3}\right) \text {. }
\end{aligned}
$$

Thus, substituting (22) into (21), we have

$$
\begin{aligned}
& \left(\left\|\varphi_{x}^{n+1 / 2}\right\|^{2}-\left\|\varphi_{x}^{n-1 / 2}\right\|^{2}\right)+\frac{1}{2}\left(\left\|u^{n+1}\right\|^{2}-\left\|u^{n-1}\right\|^{2}\right) \\
& +\left(\left\|\varphi_{x x}^{n+1 / 2}\right\|^{2}-\left\|\varphi_{x x}^{n-1 / 2}\right\|^{2}\right) \\
& +\frac{1}{2}\left(\left\|u_{x}^{n+1}\right\|^{2}-\left\|u_{x}^{n-1}\right\|^{2}\right) \\
& +\left(\left\|\varphi_{x x x}^{n+1 / 2}\right\|^{2}-\left\|\varphi_{x x x}^{n-1 / 2}\right\|^{2}\right) \\
& +\frac{h}{3} \sum_{j=1}^{J}\left(\left(u_{j}^{n+1}\right)^{3}-\left(u_{j}^{n-1}\right)^{3}\right)=0
\end{aligned}
$$

so that

$$
\begin{aligned}
\frac{1}{2}\left(\left\|u^{n+1}\right\|^{2}+\left\|u^{n}\right\|^{2}+\left\|u_{x}^{n+1}\right\|^{2}+\left\|u_{x}^{2}\right\|^{2}\right)+\left\|\varphi_{x}^{n+1 / 2}\right\|^{2} \\
+\left\|\varphi_{x x}^{n+1 / 2}\right\|^{2}+\left\|\varphi_{x x x}^{n+1 / 2}\right\|^{2} \\
+\frac{h}{3} \sum_{j=1}^{J}\left[\left(u_{j}^{n+1}\right)^{3}+\left(u_{j}^{n}\right)^{3}\right] \\
=\frac{1}{2}\left(\left\|u^{n}\right\|^{2}+\left\|u^{n-1}\right\|^{2}+\left\|u_{x}^{n}\right\|^{2}+\left\|u_{x}^{n-1}\right\|^{2}\right) \\
+\left\|\varphi_{x}^{n-\frac{1}{2}}\right\|^{2}+\left\|\varphi_{x x}^{n-\frac{1}{2}}\right\|^{2}+\left\|\varphi_{x x x}^{n-\frac{1}{2}}\right\|^{2} \\
+\frac{h}{3} \sum_{j=1}^{J}\left(\left(u_{j}^{n}\right)^{3}+\left(u_{j}^{n-1}\right)^{3}\right) ;
\end{aligned}
$$

we let

$$
\begin{aligned}
E^{n}= & \frac{1}{2}\left(\left\|u^{n+1}\right\|^{2}+\left\|u^{n}\right\|^{2}+\left\|u_{x}^{n+1}\right\|^{2}+\left\|u_{x}^{n}\right\|^{2}\right) \\
& +\left\|\varphi_{x}^{n+\frac{1}{2}}\right\|^{2}+\left\|\begin{array}{c}
n+\frac{1}{2} \|^{2} \\
\varphi_{x x}
\end{array}+\right\| \begin{array}{c}
n+\frac{1}{2} \\
\varphi_{x x x}^{2}
\end{array} \\
& +\frac{1}{3} \sum_{j=1}^{J}\left(\left(u_{j}^{n+1}\right)^{3}+\left(u_{j}^{n}\right)^{3}\right) .
\end{aligned}
$$

Then we obtain

$$
E^{n}=E^{n-1}=\cdots=E^{0} .
$$

The proof of Theorem 6 is completed.

\section{The Existence of the Difference Solutions}

Theorem 7. There exists $u^{n} \in Z_{h}^{0}$ which satisfies the difference scheme (11). 
Discrete Dynamics in Nature and Society

5

Proof. We assume that when $n<N$, there exist $u^{0}, u^{1}, u^{2}$, $\ldots, u^{n}$ satisfying (11). Next we prove that there exists $u^{n+1}$ which satisfies (11). Let $w(v)$ be an operator on $Z_{h}^{0}$ defined by

$$
\begin{aligned}
& w(v) \\
& =\frac{1}{\tau^{2}}\left(v-2 u^{n}+2 u^{n-1}\right)-\frac{1}{2}\left(v+2 u^{n-1}\right)_{x \bar{x}} \\
& \quad+\frac{1}{2}\left(v+2 u^{n-1}\right)_{x x \bar{x} \bar{x}}-\frac{1}{\tau^{2}}\left(v_{x \bar{x}}-2 u_{x \bar{x}}^{n}+2 u_{x \bar{x}}^{n-1}\right) \\
& +\frac{1}{\tau^{2}}\left(v_{x x \bar{x} \bar{x}}-2 u_{x x \bar{x} \bar{x}}^{n}+2 u_{x x \bar{x} \bar{x}}^{n-1}\right) \\
& \quad-\frac{1}{3}\left(\left(v+u^{n-1}\right)^{2}+\left(u^{n-1}\right)^{2}+\left(v+u^{n-1}\right)\left(u^{n-1}\right)\right)
\end{aligned}
$$

obviously $w(v)$ is continuous. Computing the inner product of (27) with $v$, we obtain

$$
\langle w(v), v\rangle=\mathrm{I}^{\prime}-\mathrm{II}^{\prime}+\mathrm{III}^{\prime}-\mathrm{IV}^{\prime}-\mathrm{V}^{\prime}-\mathrm{VI}^{\prime},
$$

where

$$
\begin{aligned}
\mathrm{I}^{\prime} & =\frac{1}{\tau^{2}}\left\langle\left(v-2 u^{n}+2 u^{n-1}\right), v\right\rangle=\frac{1}{\tau^{2}}\left(\| v-u^{n}\right. \\
& \left.+u^{n-1}\left\|^{2}-\right\| u^{n}-u^{n-1} \|^{2}\right), \\
\mathrm{II}^{\prime} & =\frac{1}{2}\left\langle\left(v+2 u^{n-1}\right)_{x \bar{x}}, v\right\rangle=-\frac{1}{2}\left(\left\|v_{x}+u_{x}^{n-1}\right\|^{2}\right. \\
& \left.-\left\|u_{x}^{n-1}\right\|^{2}\right), \\
\mathrm{III}^{\prime} & =\frac{1}{2}\left\langle\left(v+2 u^{n-1}\right)_{x x \bar{x} \bar{x}}, v\right\rangle=\frac{1}{2}\left(\left\|v_{x x}+u_{x x}^{n-1}\right\|^{2}\right. \\
& \left.-\left\|u_{x x}^{n-1}\right\|^{2}\right), \\
& \left.+\left\|u^{n-1}\right\|_{4}^{4}+\left\|v+u^{n-1}\right\|^{2}+\left\|u^{n-1}\right\|^{2}\right) ; \\
\mathrm{VI}^{\prime} & =\frac{1}{\tau^{2}}\left\langle\left(v-2 u^{n}+2 u^{n-1}\right)_{x \bar{x}}, v\right\rangle=-\frac{1}{\tau^{2}}\left(\| v_{x}-u_{x}^{n}\right. \\
& \left.+u_{x}^{n-1}\left\|^{2}-\right\| u_{x}^{n}-u_{x}^{n-1} \|^{2}\right), \\
\mathrm{V}^{\prime} & =\frac{1}{\tau^{2}}\left\langle\left(v-2 u^{n}+2 u^{n-1}\right)_{x x \bar{x} \bar{x}}, v\right\rangle=\frac{1}{\tau^{2}}\left(\| v_{x x}\right.
\end{aligned}
$$

therefore

$$
\begin{aligned}
& (w(v), v) \geq \frac{1}{\tau^{2}}\left(\left\|v-u^{n}+u^{n-1}\right\|^{2}-\left\|u^{n}-u^{n-1}\right\|^{2}\right. \\
& +\left\|v_{x}-u_{x}^{n}+u_{x}^{n-1}\right\|^{2}-\left\|u_{x}^{n}-u_{x}^{n-1}\right\|^{2} \\
& \left.+\left\|v_{x x}-u_{x x}^{n}+u_{x x}^{n-1}\right\|^{2}-\left\|u_{x x}^{n}-u_{x x}^{n-1}\right\|^{2}\right) \\
& +\frac{1}{2}\left(\left\|v_{x}+u_{x}^{n-1}\right\|^{2}-\left\|u_{x}^{n-1}\right\|^{2}+\left\|v_{x x}+u_{x x}^{n-1}\right\|^{2}\right. \\
& \left.-\left\|u_{x x}^{n-1}\right\|^{2}\right)-\frac{1}{6}\left\|v+u^{n-1}\right\|_{4}^{4}-\frac{1}{6}\left\|u^{n-1}\right\|_{4}^{4}-\frac{1}{6} \| v \\
& +u^{n-1}\left\|^{2}-\frac{1}{6}\right\| u^{n-1}\left\|^{2} \geq \frac{1}{\tau^{2}}\right\| v-u^{n}+u^{n-1} \|^{2} \\
& +\left(\frac{1}{\tau^{2}}\left\|u^{n}-u^{n-1}\right\|^{2}+\frac{1}{\tau^{2}}\left\|u_{x}^{n}-u_{x}^{n-1}\right\|^{2}\right. \\
& +\frac{1}{\tau^{2}}\left\|u_{x x}^{n}-u_{x x}^{n-1}\right\|^{2}+\frac{1}{2}\left\|u_{x}^{n-1}\right\|^{2}+\frac{1}{2}\left\|u_{x x}^{n-1}\right\|^{2} \\
& +\frac{1}{6}\left\|v+u^{n-1}\right\|_{4}^{4}+\frac{1}{6}\left\|u^{n-1}\right\|_{4}^{4}+\frac{1}{6}\left\|v+u^{n-1}\right\|^{2} \\
& \left.+\frac{1}{6}\right)
\end{aligned}
$$

where

$$
\left\|v-u^{n}+u^{n-1}\right\|^{2} \geq \frac{1}{2}\|v\|^{2}-2\left\|u^{n}-u^{n-1}\right\|^{2}
$$

then

$$
\begin{aligned}
& (w(v), v) \geq \frac{1}{2 \tau^{2}}\|v\|^{2}-\left(\frac{3}{\tau^{2}}\left\|u^{n}-u^{n-1}\right\|^{2}\right. \\
& +\frac{1}{\tau^{2}}\left\|u_{x}^{n}-u_{x}^{n-1}\right\|^{2}+\frac{1}{\tau^{2}}\left\|u_{x x}^{n}-u_{x x}^{n-1}\right\|^{2}+\frac{1}{2}\left\|u_{x}^{n-1}\right\|^{2} \\
& +\frac{1}{2}\left\|u_{x x}^{n-1}\right\|^{2}+\frac{1}{6}\left\|v+u^{n-1}\right\|_{4}^{4}+\frac{1}{6}\left\|u^{n-1}\right\|_{4}^{4} \\
& \left.+\frac{1}{6}\left\|v+u^{n-1}\right\|^{2}+\frac{1}{6}\left\|u^{n-1}\right\|^{2}\right)
\end{aligned}
$$

Hence it is obvious that $(w(v), v)>1 \forall v \in Z_{h}^{0}$ with $\|v\|^{2}=$ $6\left\|u^{n}-u^{n-1}\right\|^{2}+2\left\|u_{x}^{n}-u_{x}^{n-1}\right\|^{2}+2\left\|u_{x x}^{n}-u_{x x}^{n-1}\right\|^{2}+2 \tau^{2}\left\|u_{x}^{n-1}\right\|^{2}+$ $2 \tau^{2}\left\|u_{x x}^{n-1}\right\|^{2}+\left(\tau^{2} / 3\right)\left\|v+u^{n-1}\right\|_{4}^{4}+\left(\tau^{2} / 3\right)\left\|u^{n-1}\right\|_{4}^{4}+\left(\tau^{2} / 3\right) \| v+$ $u^{n-1}\left\|^{2}+\left(\tau^{2} / 3\right)\right\| u^{n-1} \|^{2}+1$. It follows from Lemma 5 that there exists $v^{*} \in Z_{h}^{0}$ such that $w\left(v^{*}\right)=0$. If we take $u^{n+1}=v^{*}+u^{n-1}$, it satisfies (11). This completes the proof. 


\section{Convergence and Stability}

Let the solution of problem (1)-(3) be $\widetilde{u}(x, t)$; that is, $\tilde{u}_{j}^{n}=$ $\widetilde{u}\left(x_{j}, t_{n}\right)$. Then we consider the truncation error of the difference scheme of (11)-(15) as follows:

$$
\begin{aligned}
r_{j}^{n}= & \left(\tilde{u}_{j}^{n}\right)_{t \bar{t}}-\left(\overline{\bar{u}}_{j}^{n}\right)_{x \bar{x}}-\left(\tilde{u}_{j}^{n}\right)_{x \bar{x} t \bar{t}}+\left(\overline{\tilde{u}}_{j}^{n}\right)_{x x \bar{x} \bar{x}} \\
& +\left(\widetilde{u}_{j}^{n}\right)_{x x \bar{x} \bar{x} t \bar{t}} \\
& +\frac{1}{3}\left(\left(\widetilde{u}_{j}^{n+1}\right)^{2}+\left(\widetilde{u}_{j}^{n+1}\right)\left(\tilde{u}_{j}^{n-1}\right)+\left(\widetilde{u}_{j}^{n-1}\right)^{2}\right)_{x \bar{x}} .
\end{aligned}
$$

According to Taylor expansion, we obtain that when $h, \tau \rightarrow 0$, $r_{j}^{n}=O\left(h^{2}+\tau^{2}\right)$.

Theorem 8. The solution of the finite difference scheme (11)-(15) converges to the solution of the initial-boundary value problem (1)-(3) with order $O\left(h^{2}+\tau^{2}\right)$ by the $L_{\infty}$ norm.

Proof. We define the solution of the finite difference scheme (11)-(15) which is $u_{j}^{n}$. Let $e_{j}^{n}=\widetilde{u}_{j}^{n}-u_{j}^{n}$ and $\left(e_{j}^{n}\right)_{t}=\left(\delta_{j}^{n+1 / 2}\right)_{x \bar{x}}$. Subtracting (33) to (11), we have

$$
\begin{aligned}
r_{j}^{n}= & \left(e_{j}^{n}\right)_{t \bar{t}}-\left(\bar{e}_{j}^{n}\right)_{x \bar{x}}-\left(e_{j}^{n}\right)_{x \bar{x} t \bar{t}}+\left(\bar{e}_{j}^{n}\right)_{x x \bar{x} \bar{x}} \\
& +\left(e_{j}^{n}\right)_{x x \bar{x} \bar{x} t \bar{t}}+\left(W_{j}\right)_{x \bar{x}},
\end{aligned}
$$

where

$$
\begin{aligned}
W_{j}= & \frac{1}{3}\left(\left(\widetilde{u}_{j}^{n+1}\right)^{2}+\left(\widetilde{u}_{j}^{n+1}\right)\left(\widetilde{u}_{j}^{n-1}\right)+\left(\widetilde{u}_{j}^{n-1}\right)^{2}\right) \\
& -\frac{1}{3}\left(\left(u_{j}^{n+1}\right)^{2}+\left(u_{j}^{n+1}\right)\left(u_{j}^{n-1}\right)+\left(u_{j}^{n-1}\right)^{2}\right) .
\end{aligned}
$$

Computing the inner product of (34) with $h\left(\delta^{n+1 / 2}+\delta^{n-1 / 2}\right)$, we get

$$
\begin{aligned}
- & \left\langle r^{n}, h\left(\delta^{n+1 / 2}+\delta^{n-1 / 2}\right)\right\rangle \\
& -\left\langle W_{x \bar{x}}, h\left(\delta^{n+1 / 2}+\delta^{n-1 / 2}\right)\right\rangle \\
= & -\mathrm{I}^{\prime \prime}+\mathrm{II}^{\prime \prime}+\mathrm{III}^{\prime \prime}-\mathrm{IV}^{\prime \prime},
\end{aligned}
$$

where

$$
\begin{aligned}
\mathrm{I}^{\prime \prime} & =\left\langle\left(e^{n}\right)_{t \bar{t}}, h\left(\delta^{n+1 / 2}+\delta^{n-1 / 2}\right)\right\rangle=-\frac{h}{\tau}\left(\left\|\delta^{n+1 / 2}\right\|^{2}\right. \\
& \left.-\left\|\delta^{n-1 / 2}\right\|^{2}\right), \\
\mathrm{II}^{\prime \prime} & =\left\langle\left(\bar{e}^{n}\right)_{x \bar{x}}, h\left(\delta^{n+1 / 2}+\delta^{n-1 / 2}\right)\right\rangle=\frac{h}{2 \tau}\left(\left\|e^{n+1}\right\|^{2}\right. \\
& \left.-\left\|e^{n-1}\right\|^{2}\right),
\end{aligned}
$$

$$
\begin{aligned}
\mathrm{III}^{\prime \prime} & =\left\langle\left(e^{n}\right)_{x \bar{x} \bar{t} \bar{t}}, h\left(\delta^{n+1 / 2}+\delta^{n-1 / 2}\right)\right\rangle=\frac{h}{\tau}\left(\left\|\delta_{x x}^{n+1 / 2}\right\|^{2}\right. \\
- & \left.\left\|\delta_{x x}^{n-1 / 2}\right\|^{2}\right), \\
\mathrm{IV}^{\prime \prime} & =\left\langle\left(\bar{e}^{n}\right)_{x x \bar{x} \bar{x}}, h\left(\delta^{n+1 / 2}+\delta^{n-1 / 2}\right)\right\rangle \\
& =-\frac{h}{2 \tau}\left(\left\|e_{x}^{n+1}\right\|^{2}-\left\|e_{x}^{n-1}\right\|^{2}\right), \\
\mathrm{V}^{\prime \prime} & =\left\langle\left(e^{n}\right)_{x x \bar{x} \bar{x} \bar{t}}, h\left(\delta^{n+1 / 2}+\delta^{n-1 / 2}\right)\right\rangle \\
& =-\frac{h}{\tau}\left(\left\|\delta_{x x x}^{n+1 / 2}\right\|^{2}-\left\|\delta_{x x x}^{n-1 / 2}\right\|^{2}\right), \\
\langle & \left.W_{x \bar{x}}, h\left(\delta^{n+1 / 2}+\delta^{n-1 / 2}\right)\right\rangle=\frac{h^{2}}{3 \tau} \\
\cdot & \sum_{j=1}^{J}\left(\left(\frac{\left(\widetilde{u}_{j}^{n+1}\right)^{3}-\left(\widetilde{u}_{j}^{n-1}\right)^{3}}{\widetilde{u}_{j}^{n+1}-\widetilde{u}_{j}^{n-1}}-\frac{\left(u_{j}^{n+1}\right)^{3}-\left(u_{j}^{n-1}\right)^{3}}{u_{j}^{n+1}-u_{j}^{n-1}}\right)\right.
\end{aligned}
$$$$
\left.\cdot\left(e_{j}^{n+1}-e_{j}^{n-1}\right)\right) \text {. }
$$

By Lemma 3, it can be immediately obtained that there exist $\theta \in(-1,1)$ and $\eta \in[a, b]$, such that

$$
\begin{aligned}
& \frac{h^{2}}{3 \tau} \sum_{j=1}^{J}\left(\left(\frac{\left(\widetilde{u}_{j}^{n+1}\right)^{3}-\left(\widetilde{u}_{j}^{n-1}\right)^{3}}{\widetilde{u}_{j}^{n+1}-\widetilde{u}_{j}^{n-1}}-\frac{\left(u_{j}^{n+1}\right)^{3}-\left(u_{j}^{n-1}\right)^{3}}{u_{j}^{n+1}-u_{j}^{n-1}}\right)\right. \\
& \left.\cdot\left(e_{j}^{n+1}-e_{j}^{n-1}\right)\right)=\frac{h^{2}}{3 \tau} \\
& \cdot \sum_{j=1}^{J}\left(6 \eta\left(\frac{1-\theta}{2} e_{j}^{n-1}+\frac{1+\theta}{2} e_{j}^{n+1}\right)\left(e_{j}^{n+1}-e_{j}^{n-1}\right)\right) \\
& \leq \frac{h C}{\tau}\left(\left\|e_{j}^{n+1}\right\|^{2}+\left\|e_{j}^{n-1}\right\|^{2}\right) ;
\end{aligned}
$$

furthermore

$$
\begin{aligned}
& -\left\langle r^{n}, h\left(\delta^{n+\frac{1}{2}}+\delta^{n-\frac{1}{2}}\right)\right\rangle \leq C h\left(\left\|r^{n}\right\|^{2}\right. \\
& +\left\|\delta^{n+\frac{1}{2}}\right\|^{2}+\left\|\delta^{n-\frac{1}{2}}\right\|^{2}+\left\|\delta_{x x}^{n+\frac{1}{2}}\right\|^{2}+\left\|\delta_{x x}^{n-\frac{1}{2}}\right\|^{2} \|^{2} \\
& \left.+\left\|\begin{array}{c}
n+\frac{1}{2} \\
\delta_{x x x}
\end{array}\right\|^{2}+\| \begin{array}{c}
n-\frac{1}{2} \\
\delta_{x x x}^{2}
\end{array}\right) ;
\end{aligned}
$$


combining (36) with (39), we can get

$$
\begin{aligned}
& \left\langle r^{n}, h\left(\delta^{n+1 / 2}+\delta^{n-1 / 2}\right)\right\rangle \\
& -\left\langle W_{x \bar{x}}, h\left(\delta^{n+1 / 2}+\delta^{n-1 / 2}\right)\right\rangle \leq C h\left(\left\|\delta^{n+1 / 2}\right\|^{2}\right. \\
& +\left\|\delta^{n-1 / 2}\right\|^{2}+\left\|\delta_{x x}^{n+1 / 2}\right\|^{2}+\left\|\delta_{x x}^{n-1 / 2}\right\|^{2}+\left\|\delta_{x x x}^{n+1 / 2}\right\|^{2} \\
& \left.+\left\|\delta_{x x x}^{n-1 / 2}\right\|^{2}\right)+\frac{C h}{\tau}\left(\left\|e_{j}^{n+1}\right\|^{2}+\left\|e_{j}^{n-1}\right\|^{2}\right)+C h\left\|r^{n}\right\|^{2} .
\end{aligned}
$$

Let

$$
\begin{aligned}
B^{n}= & \left\|\delta^{n+1 / 2}\right\|^{2}+\left\|\delta_{x x}^{n+1 / 2}\right\|^{2}+\left\|\delta_{x x x}^{n+1 / 2}\right\|^{2} \\
& +\frac{1}{2}\left(\left\|e^{n+1}\right\|^{2}+\left\|e^{n}\right\|^{2}\right)+\frac{1}{2}\left(\left\|e_{x}^{n+1}\right\|^{2}+\left\|e_{x}^{n}\right\|^{2}\right),
\end{aligned}
$$

so that (40) can be expressed as

$$
B^{n}-B^{n-1} \leq C \tau\left\|r^{n}\right\|^{2}+C \tau\left(B^{n}+B^{n-1}\right) ;
$$

summing (42) from 0 to $N$,

$$
B^{N} \leq B^{0}+C \tau \sum_{l=0}^{N}\left\|r_{l}^{n}\right\|^{2}+C \tau \sum_{l=0}^{N} B^{l}
$$

furthermore

$$
\begin{aligned}
\tau \sum_{l=0}^{N}\left\|r_{l}^{n}\right\|^{2} & \leq N \tau \max _{0 \leq l \leq N}\left\|r_{l}^{n}\right\|^{2} \leq T O\left(\tau^{2}+h^{2}\right)^{2}, \\
e^{0} & =0
\end{aligned}
$$

therefore

$$
B^{N} \leq O\left(\tau^{2}+h^{2}\right)^{2}+C \tau \sum_{l=0}^{N} B^{l}
$$

By Lemma 4, we can get

$$
B^{N} \leq O\left(\tau^{2}+h^{2}\right)^{2}
$$

It follows that

$$
\begin{aligned}
& \left\|e^{n}\right\| \leq O\left(\tau^{2}+h^{2}\right), \\
& \left\|e_{x}^{n}\right\| \leq O\left(\tau^{2}+h^{2}\right) .
\end{aligned}
$$

By Lemma 2, we can get

$$
\left\|e^{n}\right\|_{\infty} \leq O\left(\tau^{2}+h^{2}\right)
$$

Theorem 9. The finite difference scheme (11)-(15) is stable by the $L_{\infty}$ norm.
Proof. Let $U_{j}^{n}$ be another solution of the finite difference scheme (11)-(15), so that we can have the initial conditions

$$
U_{j}^{0}=u_{0}\left(x_{j}\right)+\varepsilon_{0}\left(x_{j}\right),
$$

where $\varepsilon_{0}\left(x_{j}\right)$ is a small perturbation of initial value. Then we define the error as follows:

$$
\begin{aligned}
\widetilde{e}_{j}^{n} & =U_{j}^{n}-u_{j}^{n}, \\
\left(\widetilde{e}_{j}^{n}\right)_{t} & =\left(\widetilde{\delta}_{j}^{n+1 / 2}\right)_{x \bar{x}} .
\end{aligned}
$$

Therefore, the error satisfies the following equation:

$$
\begin{aligned}
\left(\widetilde{e}_{j}^{n}\right)_{t \bar{t}} & -\left(\overline{\tilde{e}}_{j}^{n}\right)_{x \bar{x}}-\left(\widetilde{e}_{j}^{n}\right)_{x \bar{x} t \bar{t}}+\left(\overline{\tilde{e}}_{j}^{n}\right)_{x x \bar{x} \bar{x}}+\left(\widetilde{e}_{j}^{n}\right)_{x x \bar{x} \bar{x} t \bar{t}} \\
& +\left(\widetilde{W}_{j}\right)_{x \bar{x}}=0,
\end{aligned}
$$

where

$$
\begin{aligned}
\widetilde{W}_{j}= & \frac{1}{3}\left(\left(U_{j}^{n+1}\right)^{2}+\left(U_{j}^{n+1}\right)\left(U_{j}^{n-1}\right)+\left(U_{j}^{n-1}\right)^{2}\right) \\
& -\frac{1}{3}\left(\left(u_{j}^{n+1}\right)^{2}+\left(u_{j}^{n+1}\right)\left(u_{j}^{n-1}\right)+\left(u_{j}^{n-1}\right)^{2}\right) .
\end{aligned}
$$

Similar to the proof of Theorem 8 , it is easy to get $\left\|\tilde{e}^{n}\right\|^{2} \leq$ $C\left\|U^{0}-u^{0}\right\|^{2}$. Thus the stability can be proved.

\section{Numerical Experiments}

In this section, some numerical tests are given to support the convergence order and energy conservation of the scheme proposed in this paper. We performed our experiments in Wolfram Mathematica 9.0 soft on an Intel(R) Xeon(R)E5620, $2.40 \mathrm{GHz}$ dual-core CPU PC with $8 \mathrm{~GB}$ of memory. The computational space and time convergence order of the method are calculated with the following formulas:

$$
\begin{aligned}
& \frac{\log \left(e_{1} / e_{2}\right)}{\log \left(h_{1} / h_{2}\right)}, \\
& \frac{\log \left(e_{1} / e_{2}\right)}{\log \left(\tau_{1} / \tau_{2}\right)},
\end{aligned}
$$

where $e_{i}$ are errors corresponding $\left(e=\left\|U^{N}-u^{N}\right\|_{\infty}\right)$ to mesh size $h_{i}$ and $\tau_{i}(i=1,2)$, respectively.

We consider the generalized Boussinesq equation on the region $(x, t) \in[0,2 \pi] \times[0, T]$. The initial conditions solution are given by

$$
\begin{gathered}
u(x, 0)=\sin (x), \\
u_{t}(x, 0)=2 \sin (x) .
\end{gathered}
$$

Since we do not know the exact solution of the generalized Boussinesq equation, an error estimate method has been used: a comparison between the numerical solutions on a coarse mesh and those on a refine mesh is made. We consider the solution on mesh $h=\pi / 320, \tau=0.0001$, by the scheme proposed in this paper as the reference solution. 
TABLE 1: Energy error and space convergence orders by the scheme proposed in this paper with $\tau=0.001$ and several values of $h$ for $T=1$.

\begin{tabular}{lcccc}
\hline$h$ & $L_{\infty}$ error & Order & $E(0)$ & $|E(0)-E(T)|$ \\
\hline $2 \pi / 5$ & $7.3525 \times 10^{-2}$ & $*$ & 4.779952984322570 & $8.5265 \times 10^{-14}$ \\
$2 \pi / 10$ & $1.7082 \times 10^{-2}$ & 2.1057 & 4.783077540608440 & $2.1316 \times 10^{-14}$ \\
$2 \pi / 20$ & $4.8150 \times 10^{-3}$ & 1.8269 & 4.783267130324504 & $6.3949 \times 10^{-14}$ \\
$2 \pi / 40$ & $1.1918 \times 10^{-3}$ & 2.0144 & 4.783278869330395 & $4.9738 \times 10^{-15}$ \\
$2 \pi / 80$ & $2.9391 \times 10^{-4}$ & 2.0197 & 4.783279601233270 & $8.3134 \times 10^{-13}$ \\
\hline
\end{tabular}

* There are no data.

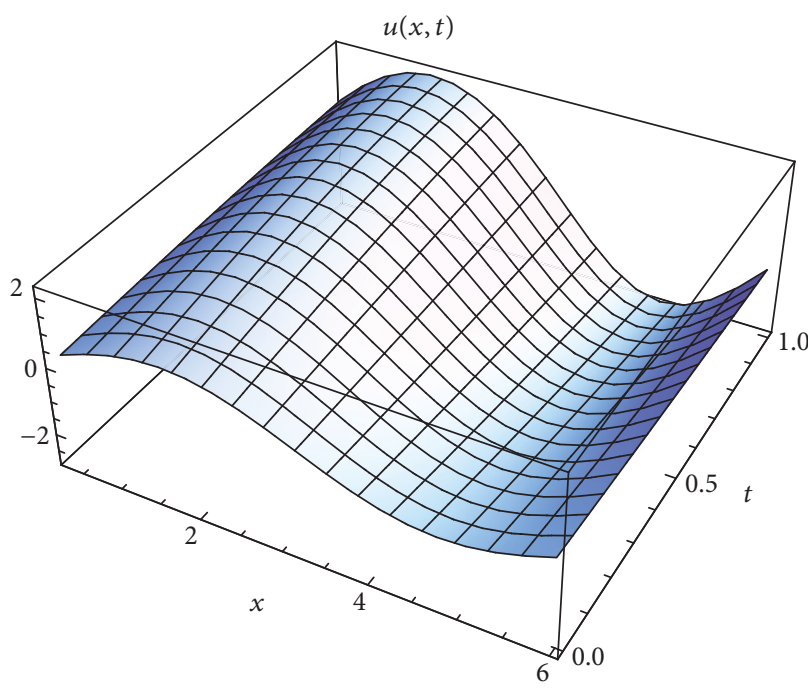

(a) Numerical solution of $u(x, t)$

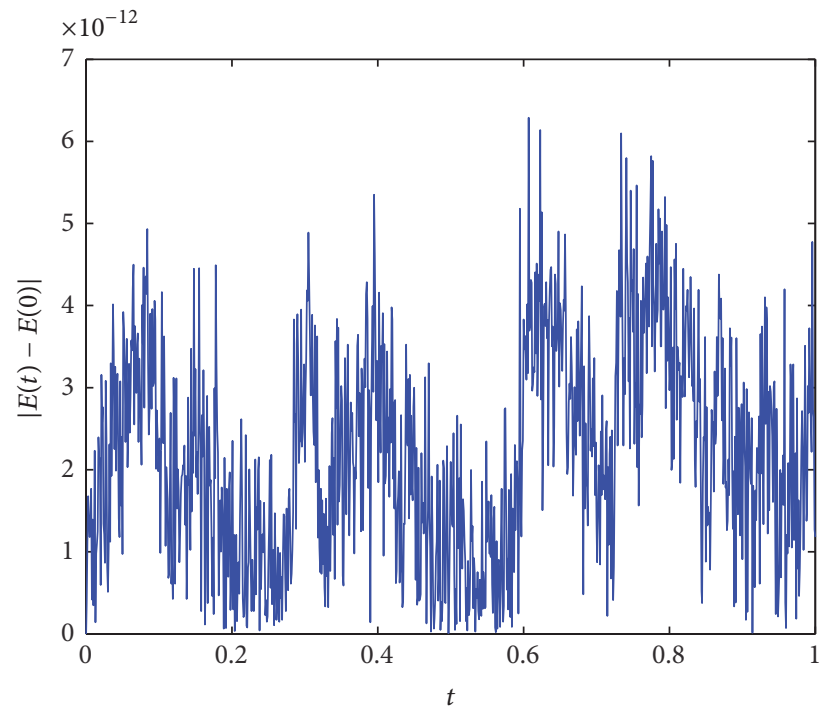

(b) Energy difference $|E(t)-E(0)|$

FIGURE 1: (a) Space-time graph of numerical solution and (b) the energy difference $|E(t)-E(0)|$ in each time level of the problem with different $h=\pi / 40, T=1$, and $\tau=0.05$.

TABLE 2: Error and computational time orders of the scheme proposed in this paper with $h=\pi / 160$ and several values of $\tau$ for $T=1$.

\begin{tabular}{lcc}
\hline$\tau$ & $L_{\infty}$ error & Order \\
\hline $1 / 18$ & $1.8975 \times 10^{-2}$ & $*$ \\
$1 / 36$ & $4.7947 \times 10^{-3}$ & 1.9846 \\
$1 / 72$ & $1.2050 \times 10^{-3}$ & 1.9925 \\
$1 / 144$ & $3.0200 \times 10^{-4}$ & 1.9964 \\
$1 / 288$ & $7.5588 \times 10^{-5}$ & 1.9983 \\
\hline
\end{tabular}

${ }^{*}$ There are no data.

The problem is solved using the scheme proposed in this paper with $\tau=0.001$ and several values of $h$ for $T=1$, and the errors, computational space orders (corresponding to $\left.L_{\infty}\right)$, the initial energy, and energy difference $|E(T)-E(0)|$ are shown in Table 1 . The numerical results in Table 1 demonstrate that the convergence of space is of order 2. Besides the conservation law of energy is verified very well for the energy difference $|E(T)-E(0)|$ in Table 1.

In Table 2, we show the $L_{\infty}$ error and computational time convergence orders of $u$ with $h=\pi / 160$ and several values of $\tau$ for $T=1$. The numerical results show that the time convergence orders of the scheme proposed in this paper are 2.

Space-time graph of numerical solution of the problem with $h=\pi / 40, T=1$, and $\tau=0.05$ is shown in Figure 1(a). The energy difference $|E(t)-E(0)|$ in each time level of test problem with different $h=\pi / 40, T=1$, and $\tau=0.05$ is shown in Figure 1(b). It can be observed from Figure 1 that the scheme proposed in this paper has good energy conservative behaviors.

\section{Conclusions}

In this paper, we have presented a conservation of finite difference scheme for the initial-boundary value problem of generalized Boussinesq equations. The uniqueness, convergence, and stability with $O\left(h^{2}+\tau^{2}\right)$ of the finite difference approximate solutions were discussed in detail. The behaviors of the proposed scheme were tested on the Bq equation and the conservation law of energy is concerned through the numerical result obtained which were found close to the energy difference. 


\section{Conflicts of Interest}

The authors declare that they have no conflicts of interest.

\section{Acknowledgments}

This work was supported by the National Natural Science Foundation of China (11071033 and 11101102), the Ph.D. StartUp Fund of Liaoning Province of China (nos. 20141137 and 20141139), the Natural Science Foundation of Liaoning Province of China (no. 20170540004), and the Liaoning BaiQianWan Talents Program (no. 2013921055).

\section{References}

[1] M. B. Abbott, H. M. Petersen, and O. Skovgaard, "On the numerical modelling of short waves in shallow water," Journal of Hydraulic Research, vol. 16, no. 3, pp. 173-204, 1978.

[2] J. H. Tao, "The numerical simulation of climbing and breaking for the waves on the coastal flat," Acta Oceanologica Sinica, vol. 6, pp. 692-700, 1984.

[3] P. A. Madsen, O. R. Sørensen, and H. A. Schäffer, "Surf zone dynamics simulated by a Boussinesq type model. Part II: Surf beat and swash oscillations for wave groups and irregular waves," Coastal Engineering, vol. 32, no. 4, pp. 289-319, 1997.

[4] D. H. Peregrine, "Long waves on a beach," Journal of Fluid Mechanics, vol. 27, no. 4, pp. 815-827, 1967.

[5] Y. Zhang and J. H Tao, "A research of the difference scheme for the two-dimensional short wave equation," Ocean Engineering, vol. 18, pp. 29-37, 2000.

[6] G. W Hong and H. S Zhang, "Nonlinear numerical calculation for waters of arbitrarily varying depth," Ocean Engineering, vol. 17, pp. 64-73, 1999.

[7] L. S Zhu and G. W Hong, "Numerical calculation for nonlinear waves in water of arbitrarily varying depth with Boussinesq equations," Ocean Engineering, vol. 18, pp. 29-37, 2000.

[8] H. El-Zoheiry, "Numerical study of the improved Boussinesq equation," Chaos, Solitons and Fractals, vol. 14, no. 3, pp. 377384, 2002.

[9] A. G. Bratsos, "A second order numerical scheme for the improved Boussinesq equation," Physics Letters. A, vol. 370, no. 2, pp. 145-147, 2007.

[10] A. G. Bratsos, "A predictor-corrector scheme for the improved Boussinesq equation," Chaos, Solitons and Fractals, vol. 40, no. 5, pp. 2083-2094, 2009.

[11] T. Wang, B. Guo, and L. Zhang, "New conservative difference schemes for a coupled nonlinear Schröinger system," Applied Mathematics and Computation, vol. 217, no. 4, pp. 1604-1619, 2010.

[12] Z. Sun, "A note on finite difference method for generalized Zakharov equations," Journal of Southeast University. English Edition. Dongnan Daxue Xuebao. Yingwen Ban, vol. 16, no. 2, pp. 84-86, 2000.

[13] H. Chang, "A conservative finite difference scheme for the Camassa-Holm equation," Numerical Mathematics. A Journal of Chinese Universities. Gaodeng Xuexiao Jisuan Shuxue Xuebao, vol. 34, no. 1, pp. 78-86, 2012. 


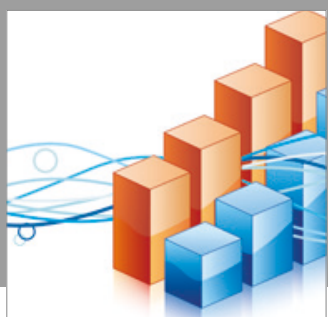

Advances in

Operations Research

vatersals

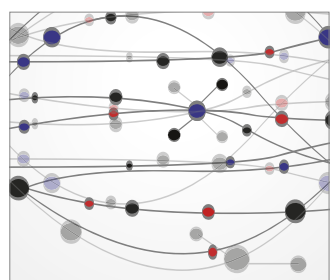

\section{The Scientific} World Journal
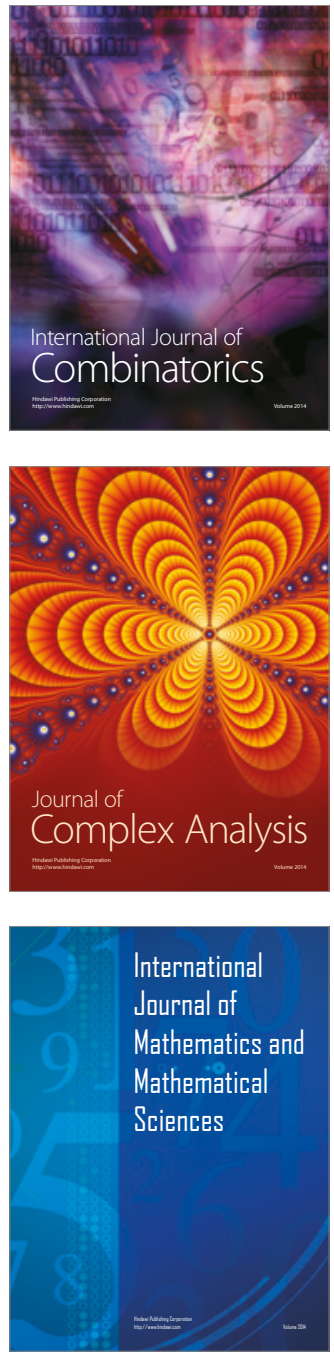
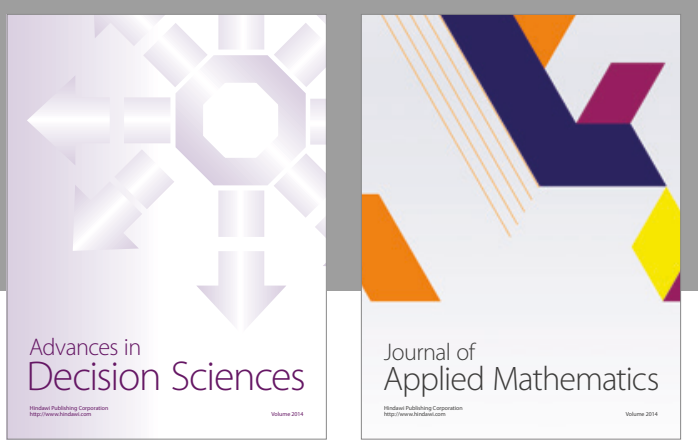

Algebra

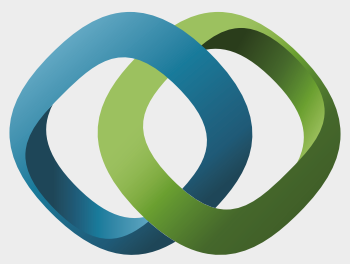

\section{Hindawi}

Submit your manuscripts at

https://www.hindawi.com
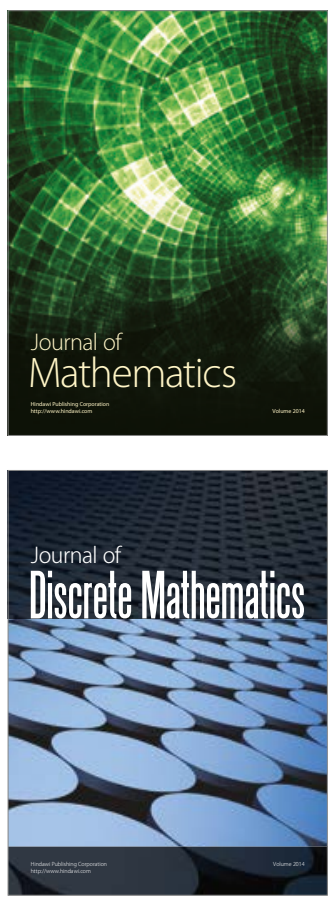

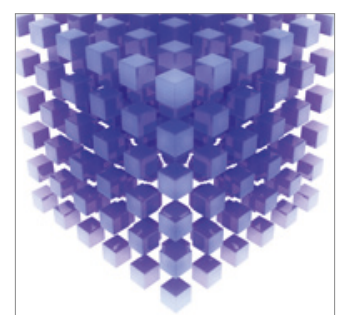

Mathematical Problems in Engineering
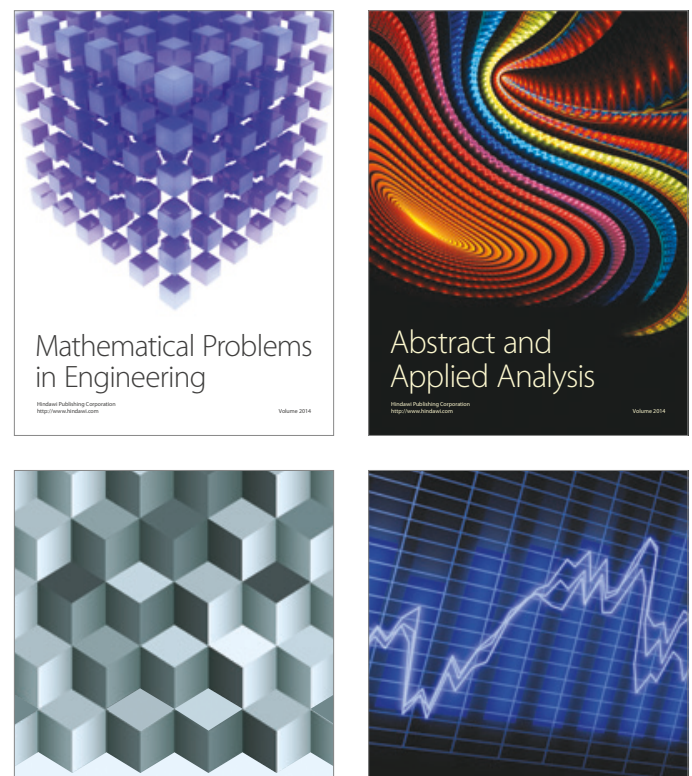

Journal of

Function Spaces

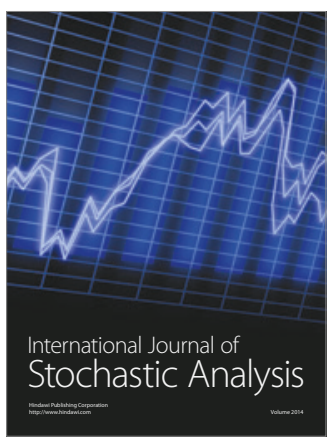

Probability and Statistics
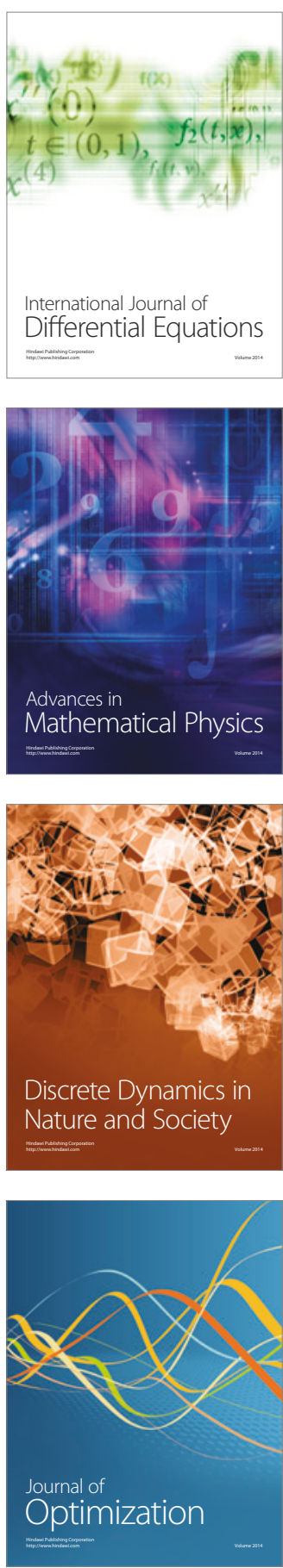